\title{
ARTICLE WITH RESPONSE: REPLY TO RESPONSE The Scaling of Speeds and Distances in Galileo's Two New Sciences: A Reply to Palmerino and Laird
}

\author{
JOHN D. NORTON* and BrYAN W. ROBERTS ${ }^{\dagger}$
}

Keywords. Acceleration, foundation of physics, freefall, Galileo, history of physics

We thank Drs. Laird and Palmerino for their efforts in responding to our analysis of Galileo's refutation of the speed-distance law of fall. We have studied their concerns. In sum, we stand by our account and the claims of our original text. We hope that readers will compare it with the critiques and come to their own conclusions. To assist them, we will remark here on some of the points they have raised.

We will respond mostly to Palmerino's comments, since hers is the more critical reaction. The locus of her concerns is the crucial first clause of Galileo's passage:

When speeds have the same ratio as the spaces passed or to be passed, those spaces come to be passed in equal times;... (Galilei, 1974, p. 160)

It contains, we urge, an ambiguity that has misdirected the literature. Do the 'speeds' and 'spaces' refer to a single moving body or to two moving bodies? Most commentators assume the single-body reading. It results in Galileo's 'very clear proof' collapsing into a circularity. We urge the two-body reading, for then the argument that follows is cogent and powerful. Of course even a thinker as adept as Galileo can commit simple fallacies. But why would we settle for that verdict on an argument that was of such importance to Galileo's narrative, when there is a second reading that exonerates him? Here, for convenience of reference, we summarize our earlier claim:

In the two-body reading of this clause, we take Galileo to be asserting a scaling result: if one takes any motion and scales up the speeds and spaces in proportion to form a new motion, the original and scaled motions occur in the same time.

Palmerino maintains that Galileo did not and could not entertain this general scaling result. Hence she concludes that 'Galileo could not have formulated the argument' we attribute to him.

\footnotetext{
*Department of History and Philosophy of Science, Center for Philosophy of Science, University of Pittsburgh, Pittsburgh, PA 15260, USA. E-mail: jdnorton@pitt.edu

†Department of History and Philosophy of Science, University of Pittsburgh, Pittsburgh, PA 15260, USA. E-mail: bwr6@pitt.edu
} 
Palmerino seeks to support this view in several ways in her critique. Setting aside remarks that locate our analysis in the literature or take up tangential disputes, we have identified four concerns in her critique that directly challenge our account and to which we will respond. She asserts:

1. Our analysis fails because we do not recognize that Galileo intends 'aggregates of speed' when he writes 'speeds' in the first sentence of his passage.

2. The scaling result we attribute to Galileo is contradicted by a special case of scaled motions derived from his mean speed theorem.

3. The treatment of scaled motion we attribute to Galileo fits poorly with those appearing in Galileo's discussion of inclined planes.

4. The geometric representation we offer of the speed-distance law of fall is an inadequate representation of the motion.

We take these concerns in turn and explain why we believe none compromises our analysis.

\section{Aggregates of Speed}

Palmerino asserts that we have misread Galileo's use of the term 'speeds,' in that we

... do not give a clear answer to the question whether they consider the term 'speeds' to indicate the final speeds of the two motions, the speeds acquired at corresponding points of the two motions, or the aggregates of all the degrees of speed acquired through the entire motions.

In particular, she suggests that we clarify our position with respect to Damerow et al. (1992, pp. 234-236), who take Galileo's first sentence to refer to 'overall velocity.' Palmerino seems to follow Damerow et al. (1992) in taking the same Italian word 'velocità' to refer to two distinct concepts in this single passage. On their reading, 'velocità' in the first sentence of the passage is taken to refer to 'overall velocity,' which Palmerino sometimes calls the 'aggregate of all the degrees of speed,' while in the remainder of the passage it is taken to refer to 'degree of velocity.' Palmerino suggests that she supports this reading, stating that the conclusions of Damerow et al . (1992) about the term 'velocità' in Galileo's passage are drawn 'correctly,' and later that,

[i]n my view, Norton and Roberts' reconstruction of Galileo's argument can only work under the assumption that in the [first] sentence ... the term 'speeds' refers to two aggregates of infinite degrees of speed.

On the contrary, we affirm what we believe is already clear both in our original text and figures: we take the term 'speeds' in this first clause to refer to the speeds acquired at the infinitely many, corresponding points of the two motions. It includes the final speed acquired at the end of the motion. It does not refer to an aggregate or overall speed. ${ }^{1}$ 
We continue to maintain that this is a plausible reading of Galileo's text. There are occasions in the Two New Sciences in which Galileo explicitly distinguishes 'somma del moto' (referred to by Palmerino sometimes as 'aggregate of all the degrees of speed' and sometimes as 'overall speed') from 'grado di velocità' ('degree of speed'). However, this passage is not one of those occasions. Galileo's passage mentions only 'velocità,' making no explicit mention of degrees or of aggregates. We interpret 'velocità' as simply meaning 'speeds,' and not as an elliptic reference to 'aggregates of speeds' as Palmerino would prefer. Indeed, this very point is a strength of our reading. Unlike Damerow et al. (1992), our reading takes 'velocità' to mean the same concept in all instances in the passage.

We do not here wish to comment on how 'aggregates of speed' entered into Galileo's thinking about freefall behind the scenes. Our analysis does not require the notion of 'aggregates of speed.' We believe that we have successfully reconstructed the steps of Galileo's argument against the speed-distance law of fall without it. The analysis succeeds by taking the word 'velocità' just to mean 'speeds' and not sometimes, covertly, 'aggregates of speeds.'

\section{Scaling Accelerated Motions by the Mean Speed Theorem}

In her second concern, Palmerino questions the compatibility of the scaling result with Galileo's mean speed theorem, in which Galileo relates uniform motion and uniformly accelerated motion. She asserts that we can know from his theorem how he would scale one particular accelerated motion, namely that which results from the speed-time law of fall. She describes a procedure for scaling this motion and finds results that, she says, are 'at odds' with our scaling result.

We make no claim concerning whether Galileo would have used the procedure she outlines. However, we do make a claim concerning the motion scaled by Palmerino's procedure: in direct contradiction with her assertion, it does conform to the scaling result.

Here is her procedure. We start with Galileo's figure from his proof of the mean speed theorem, reproduced on the left of Figure 1 below. It represents a uniform motion with the rectangle AGFB, in which the vertical line $\mathrm{AB}$ represents time and the horizontal lines represent speed, together with a corresponding uniformly accelerated motion represented by the triangle AEB. We scale up the motion by doubling all the speeds. The scaled up uniform motion, shown on the right in Figure 1, is represented by a new rectangle $\mathrm{AG}^{\prime} \mathrm{F}^{\prime} \mathrm{B}$, where $\mathrm{AG}^{\prime}$ and $\mathrm{BF}^{\prime}$ are twice the length of $\mathrm{AG}$ and $\mathrm{BF}$. The correspondingly enlarged triangle $\mathrm{AE}^{\prime} \mathrm{B}$ represents the scaled up uniformly accelerated motion.

Since the scaled up uniform motion has double the speed of the original uniform motion, it follows from the properties of uniform motion that the scaled up uniform motion $\mathrm{AG}^{\prime} \mathrm{F}^{\prime} \mathrm{B}$ covers twice the distance of the original uniform motion AGFB. We also know that the scaled up uniformly accelerated motion $\mathrm{AE}^{\prime} \mathrm{B}$ covers the same distance as the scaled up uniform motion $\mathrm{AG}^{\prime} \mathrm{F}^{\prime} \mathrm{B}$. Hence the scaled up uniformly accelerated 

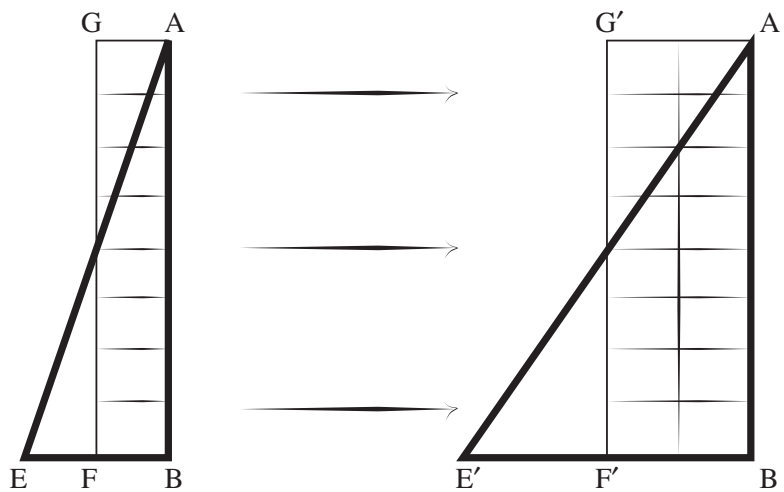

Fig. 1. Palmerino's scaling procedure. The vertical lines represent time, and the horizontal lines represent speeds.

motion passes twice the distance of the original uniformly accelerating motion. Finally, all the motions happen in the same time, since they all share the same vertical line AB. Combining, we conclude that, after the same time $\mathrm{AB}$, the original accelerated motion has acquired a final speed EB after falling some distance, and the scaled up accelerated motion has acquired twice that speed, $\mathrm{EB}^{\prime}$, after falling twice the distance.

The same result holds for any of the intermediate degrees of speed, such as the speeds acquired at the intermediate stages of motion indicated by the times $X_{1}, X_{2}, X_{3}, \ldots$ in Figure 2 below.

The uniformly accelerated motion up to those stages is indicated by the triangles $A X_{1} Y_{1}, A X_{2} Y_{2}, A X_{3} Y_{3}, \ldots$ The same analysis can be given for each of these intermediate motions individually. If we double the speeds to generate triangles $\mathrm{AX}_{1} \mathrm{Y}_{1}^{\prime}$, $\mathrm{AX}_{2} \mathrm{Y}_{2}^{\prime}, \mathrm{AX}_{3} \mathrm{Y}_{3}^{\prime}, \ldots$, then the scaled up accelerated motion covers a doubled distance
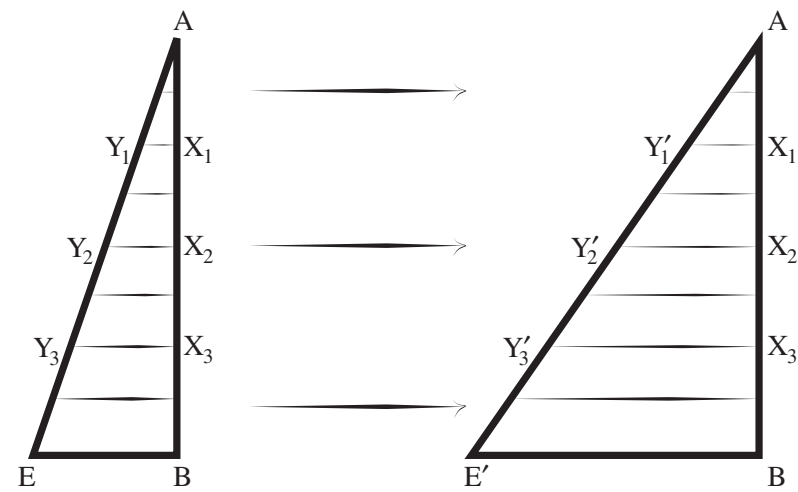

Fig. 2. Palmerino's scaling procedure for each intermediate stage of fall. 
in arriving at each stage $\mathrm{X}_{1}, \mathrm{X}_{2}, \mathrm{X}_{3}, \ldots$ and takes the same times $\mathrm{AX}_{1}, \mathrm{AX}_{2}, \mathrm{AX}_{3}, \ldots$ to arrive at them as in the original motion.

In sum, if we scale the particular case of motion governed by a speed-time law according to Palmerino's procedure, we obtain scaled motions in which all the speeds and distances conform to the scaling result we describe: when the speeds and distances are doubled for each stage of the motion, the original and the doubled motions take place in the same time.

It is worth noting, in relation to Palmerino's first concern, that this demonstration of an instance of the general scaling result has been carried out without any explicit mention of 'aggregates of speeds.'

We can calculate ${ }^{2}$ a numerical case of how the original and the scaled motions will appear according to Palmerino's procedure. The result is in the table below.

\begin{tabular}{lllrrr}
\hline & Time & 1 & 2 & 3 & 4 \\
\hline Original motion & Speed & 1 & 2 & 3 & 4 \\
& Distance & 1 & 4 & 9 & 16 \\
Palmerino's Scaled motion & Speed & 2 & 4 & 6 & 8 \\
& Distance & 2 & 8 & 18 & 32 \\
\hline
\end{tabular}

The table shows that these motions conform to our scaling result. For example, the original motion acquires speed 2 at distance 4 . The corresponding portion of the scaled up motion acquires speed 4 at distance 8 . Both portions require the same time, two units. The other three columns display the analogous relations.

Conformity of these motions with the scaling result is thereby affirmed without too much trouble. Yet Palmerino appears to have come to a different conclusion. She writes:

It must be clear, however, that the result we have just obtained is at odds with that depicted by Norton and Roberts in [her] figure 2 (=figure 1 of their article).

It is 'at odds,' she continues to explain, because the motion in our figure does not conform to the speed-time law of fall. That motion's characteristic property, Galileo's mean speed theorem ('Theorem 1, Proposition 1'), requires that 'spaces traversed are to each other as the squares of the times' and she notes correctly that the motion depicted in our figure violates this condition.

The difficulty is dissolved immediately by recalling that our figure represents the special case of motion under the speed-distance law of fall, not under the speed-time law. The scaling result is a general result that holds for all motions. It will hold if we scale motion conforming to the speed-distance law and it will hold if we scale motion conforming to the speed-time law. The resulting motions will differ in the details since they differ in the law of fall to which they conform. That is the difference Palmerino has noted. But they both conform to the scaling result. 
(a)

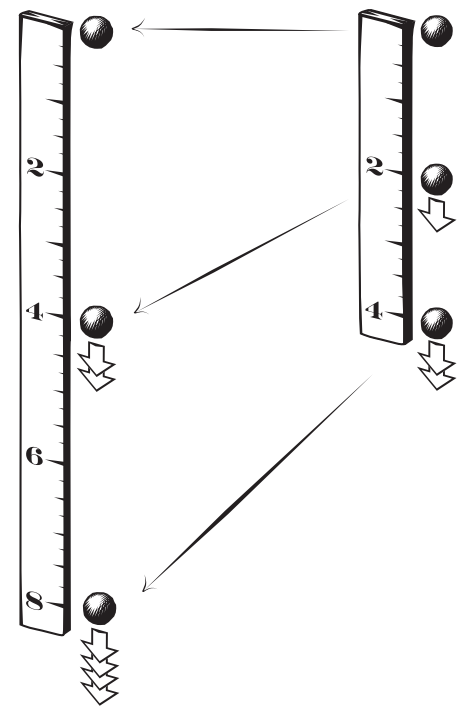

(b)

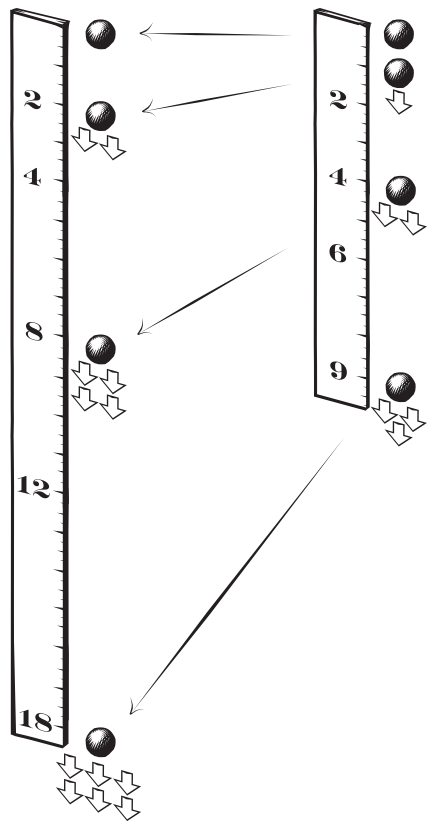

Fig. 3. Scaling of motions of the speed-distance law (a) and the speed-time law (b). The former exhibits self-similarity, while the latter does not.

To illustrate the difference in the details of scaling with respect to the two laws of fall, we reproduce our original figure on the left of Figure 3. On the right, we show the corresponding figure for the case of motion conforming to the speed-time law of fall, where the numerical values from the table above are used.

This figure once again illustrates the property distinctive of motion under the speeddistance law of fall that is essential to the analysis. In the case on the left, the scaled up motion is both a scaling of the original motion and an extension of it, so that the motion is self-similar. That self-similarity supports Galileo's conclusion that all distances are covered in the same time. This same conclusion is not available for motion under the speed-time law. For that case, the scaled up motion is a scaling of the original motion, but not an extension of it, so self-similarity fails.

\section{Scaled Motions Elsewhere}

Palmerino's third concern pertains to examples of scaled motions on inclined planes elsewhere in the text of the Third Day. She points out that these other examples differ in various details from the specifics of the scaling of motion under the speed-distance law. For example, she notes that the motions scaled in Proposition VI, Theorem VI 
are completed in the same time, but that one is not contained in the other, so that self-similarity fails.

Our response is that we should not expect these other cases to agree in all details with the scaling of motion under the speed-distance law. For, once again, these other cases are of motions that conform to the speed-time law. Indeed, they must scale differently from motions conforming to the speed-distance law. Otherwise, they too would prove objectionable. Had the motions of Proposition VI, Theorem VI proved to be self-similar, Galileo would have had to judge them as 'false and impossible...'

At a more general level, we find these last two concerns perplexing. They arise from the fallacy of applying Galileo's mean speed theorem to motion governed by a speeddistance law, whereas it is properly applied only to motion governed by a speed-time law. Are the concerns based on the unsustainable belief that Galileo must commit this fallacy? Perhaps this belief is the final import of Palmerino's remark: 'I think it is reasonable to assume that if he decided to scale up this [naturally accelerated] motion he would do it according to a natural pattern, which means that he would compare motions along differently inclined planes.'

\section{No Geometric Representation of the Speed-Distance Law}

Palmerino's fourth concern is that Galileo's treatment of motion under the speed-distance law of fall in a manuscript dated around 1604-1605 (Galileo Manuscript 72, Folio 128r) differs from the one we offer. We knew this and we discounted this difference for the reasons Palmerino mentions. Galileo's 1604-1605 analysis contains what she calls two 'mistakes.' We read Galileo in the later Two New Sciences passage as having stated the 'correct' analysis. So we infer that sometime in the three decades between 1604 and 1605 and the publication of Two New Sciences in 1638, Galileo found and corrected the mistakes.

In the context of discussing this manuscript, Palmerino makes the extraordinary claim that it is 'impossible to produce a [geometric] representation of the speed-distance law' and that Galileo was aware of this. Or, a few paragraphs later, that it is erroneous to claim ... that it is possible to offer a "similar representation" of the speed-distance law and of the speed-time law.'

This claim is surprising initially precisely because the figure in this manuscript page, reproduced as her Figure 4, is just such a representation. Galileo states in the text next to the diagram that the vertical line $\mathrm{AB}$ represents the distance that a falling body travels, while the horizontal lines represent its speed (velocità) at a given distance in fall.

The failing, according to Palmerino, is that this figure 'do[es] not show the relation between the spaces traversed and the times elapsed.' As she notes through her Figure 5, Galileo does display a figure in Two New Sciences for the speed-time law in which speeds and times are represented by the sides of a triangle and distances by rectangles. 


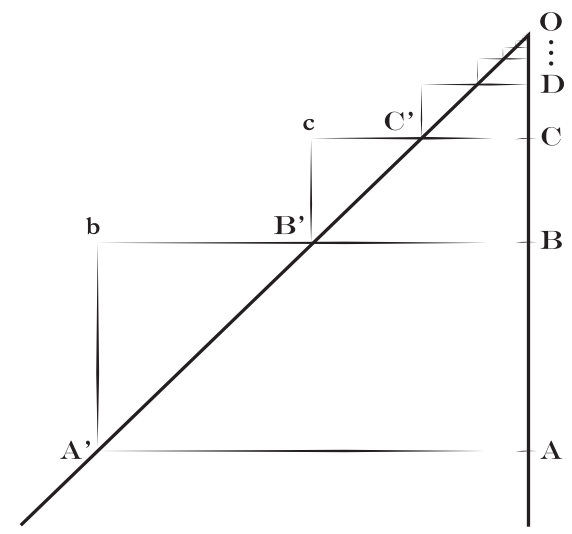

Fig. 4. Infinite time of motion in freefall under the speed-distance law.

Palmerino is correct that figures such as our original Figure 5 depicting motion under the speed-distance law do not represent time. That does not mean they are incorrect. It just means that they are incomplete. That incompleteness is far from establishing that the speed-distance law cannot be represented geometrically. No geometrical figure is able to represent exhaustively every physical aspect of some real system. All we can require is that the figure represents aspects of importance to Galileo's analysis. In this regard, Figure 5 of our original text succeeds. For the figure represents the proportionality of speed and distance according to the speed-distance law by its triangular shape. The decisive property of the motion, its self-similarity, is represented by the geometric similarity of the larger triangle $\mathrm{OCC}^{\prime}$ with small triangles that are its parts, $\mathrm{OBB}^{\prime}$ and $\mathrm{OAA}^{\prime}$. We then know from the general scaling result that the distances $\mathrm{OA}, \mathrm{OB}$ and OC are covered in the same time, which supports Galileo's dismissal of the motion.

In sum, the geometric diagram we offer is not erroneous in the sense that it misrepresents any aspect of the motion; and the diagram does offer an especially simple geometric representation of the self-similarity that is key to the entire analysis. We find no fault in it.

We are concerned that Palmerino is underestimating the expressive analytic power of the geometric methods available to Galileo. To illustrate that power, Figure 4 displays the speeds and distances of motion under the speed-distance law and also displays the infinite time needed for the falling body to complete its motion. ${ }^{3}$ We do not attribute this figure to Galileo. Indeed this figure depicts precisely the result that we claim eluded him. It employs an insight provided by Fermat (1894 [1646?]) shortly after Galileo's publication, that the time of fall according to the speed distance law is bounded below by the time required for a set of infinitely many uniform motions to occur.

The vertical line OA represents distance; $\mathrm{B}$ bisects $\mathrm{OA}$; $\mathrm{C}$ bisects $\mathrm{OB}$; D bisects OC; and so on for infinitely many bisectors. The speeds at distances $\mathrm{A}, \mathrm{B}, \mathrm{C}, \ldots$ are 
represented by horizontal lines $\mathrm{AA}^{\prime}, \mathrm{BB}^{\prime}, \mathrm{CC}^{\prime} \ldots$ and the resulting figure is a triangle since the motion is governed by the speed-distance law of fall.

The rectangle $\mathrm{AA}^{\prime} \mathrm{bB}$ represents a uniform motion over the space $\mathrm{AB}$ whose speed equals that acquired at the fastest end of the accelerated motion over the space $\mathrm{AB}$. Hence the uniform motion $\mathrm{AA}^{\prime} \mathrm{bB}$ is completed in less time than that required by the accelerated motion $\mathrm{AA}^{\prime} \mathrm{B}^{\prime} \mathrm{B}$ to cover the same space $\mathrm{AB}$. Analogously the uniform motion $\mathrm{BB}^{\prime} \mathrm{cC}$ is completed in less time than the accelerated motion $\mathrm{BB}^{\prime} \mathrm{C}^{\prime} \mathrm{C}$; and so on for the remaining infinitely many uniform motions.

Since uniform motion $\mathrm{AA}^{\prime} \mathrm{bB}$ is generated from uniform motion $\mathrm{BB}^{\prime} \mathrm{cC}$ by doubling the distance and speed, it follows from Galileo's Theorem II, Proposition II for uniform motion, that the two motions occur in the same time. Analogous reasoning for the remaining rectangles affirms that each of these infinitely many uniform motions occurs in the same time.

Combining, the times required for the accelerated motion to cover each of the segments $\mathrm{AB}, \mathrm{BC}, \mathrm{CD}, \ldots$ is greater than each of the corresponding times required for the uniform motions to cover each of these segments. Since these last times are the same and there are infinitely many of them, it follows that the total time for the accelerated motion to pass from $\mathrm{O}$ to $\mathrm{A}$ is infinite. Thus, if the body has fallen to position $\mathrm{A}$ now, at no finite time in the past was it at rest.

To repeat: we do not attribute this diagram to Galileo. We merely note that the infinitely many equal time rectangles displays the infinity of time required for the motion to be completed. The motion does, however, have the curious and potentially misleading property that its instantaneous speeds are everywhere non-zero, but the average speed for the whole motion over infinite time is zero.

There is little for us to add to Laird's remarks, which are largely expository. He recalls in some detail the achievements of Stillman Drake's analysis, and remarks that he sees 'no harm' in our reconstruction. There is one minor point to which we should alert readers. We noted that Galileo overlooked a motion that requires infinite time to fall from rest. Laird describes this as 'infinitely slow motion,' noting that Galileo had 'correctly identified [it] as rest.' The motion to which we refer is not correctly identified as rest. Rather, as Figure 4 shows, it is a motion that has been underway for all time and so it is never at rest.

We have one final remark. Laird sees a danger in 'attempting to express Galileo's geometrical proofs, which use only ratios and proportions, in the more powerful mathematics of functions and calculus.' Palmerino finds her interpretation to be a 'greater help than the anachronistic reconstruction of the argument which [we] provide in the appendix.' We agree that there are real dangers in using anachronistic methods in history of science. For this reason, we isolated later mathematical techniques in an appendix. They are not used in the main text and they are not required for us to give our analysis.

We have persisted in retaining that appendix, fully aware that just this sort of criticism would be leveled against us. Our reason is a concern over a different danger to history of 
science, the threat of irrelevance. Motion under the speed-distance law is troublesome even using modern methods. The law has the unexpected consequence of precluding motion following an initial state of rest, so it requires that a body at rest must forever remain so. Yet remarkably, Galileo was able to mount an analysis that reproduces essential results of the modern analysis, using only geometrical methods. If an excess of methodological purity prohibits historians from pointing out these associations, who will point them out? If no one does, history of science is threatened with irrelevance. The results of historians' efforts to understand Galileo's work will become entombed in a literature intellectually isolated from the sciences to which Galileo devoted much of his life.

\section{NOTES}

1. Palmerino contends that we conflate instantaneous and overall speed in our citation in our section 4.1 of Galileo's Proposition II, Theorem II on uniform motion. Our concern was the scaling result for the special case of uniform motion and we introduced this proposition because it entails a formulation of the scaling result for uniform motion in terms of instantaneous degrees of speed. That is, we take a body that moves at a constant speed of one unit, passing distances 1,2, 3 and 4 in times 1, 2, 3 and 4. That motion scales up to a body moving at constant speed 2 passing doubled distances. Applying Galileo's Prop. II, Thm. II to each stage individually, we infer that the scaled up motion passes the distances $2,4,6$, and 8 in the same times 1, 2, 3 and 4 .

2. To recover the numbers of the table, the unit of distance is chosen so that a body moving at unit speed for unit time traverses two units of distance. The individual entries are then recoverable from the mean speed theorem. For example, for the scaled motion, after two units of time, the body is moving at four units of speed. The distance passed is the same as that passed by a uniform motion at the mean speed of 2 over two units of time, which is eight units.

3. There is another simple diagram of the speed-distance law in which times can be associated with areas. That diagram is given in Figure 7 of our original text's appendix. Areas enclosed by a graph of inverse speed and distance (shown on the right) correspond to times. We make no claim, however, that Galileo was aware of this.

\section{REFERENCES}

Damerow, P., McLaughlin, P., Freudenthal, G. and Renn, J. (1992) Exploring the limits of preclassical mechanics (New York: Springer-Verlag).

Fermat, P. (1894) Fermat à Gassendi (1646?), in: P. Tannery and C. Henry (eds.) Oeuvres de Fermat, Vol. 2 (Paris: Gauthier-Villars et Fils), pp. 267-276.

Galilei, G. (1974) Two new sciences [Trans. Drake, S.] (Madison: University of Wisconsin Press). 